\title{
Improving the Future by Examining the Past
}

\author{
Charles P. Thacker \\ Microsoft Research
}

\begin{abstract}
During the last fifty years, the technology underlying computer systems has improved dramatically. As technology has evolved, designers have made a series of choices in the way it was applied in computers. In some cases, decisions that were made in the twentieth century make less sense in the twenty-first. Conversely, paths not taken might now be more attractive given the state of technology today, particularly in light of the limits the field is facing, such as the increasing gap between processor speed and storage access times and the difficulty of cooling today's computers.

In this talk, I'll discuss some of these choices and suggest some possible changes that might make computing better in the twenty-first century.
\end{abstract}

Categories \& Subject Descriptors: C.0 Computer Systems Organization; General.

General Term: Design. 